Trauma Berufskrankh 2007 - 9 [Suppl 2]:S118-S123 DOI 10.1007/s10039-006-1126-y

Online publiziert: 11. April 2006

(c) Springer Medizin Verlag 2006
O. E. Teebken · A. M. Pichlmaier · M. Karck · A. Haverich

Klinik für Thorax-, Herz- und Gefäßchirurgie,

Medizinische Hochschule Hannover, Hannover

\title{
Herz- und thorakale Gefäßverletzungen
}

Verletzungen der aszendierenden Aorta, des Aortenbogens, der Aorta descendens, der thorakalen Venen und des Herzens selbst sind mit einer hohen Mortalität verbunden. Die Diagnosestellung und die Behandlung müssen zeitnah erfolgen und erfordern apparative Voraussetzungen sowie herz-, thorax- und gefäßchirurgische Kenntnisse, die aufgrund der Subspezialisierung der chirurgischen Einzeldisziplinen nicht in jedem Krankenhaus vorgehalten werden können.

\section{Allgemeines}

Die Häufigkeit thorakaler Gefäß- und Herzverletzungen ist schwierig zu berechnen, da nicht alle Patienten das Krankenhaus lebend erreichen. In Kontinentaleuropa dominieren stumpfe Verletzungen des Thorax, die Häufigkeit penetrierender Thoraxverletzungen hat jedoch in den vergangenen Jahren immer mehr zugenommen. Die Inzidenz von interventionsbedürftigen Herz- und Gefäßverletzungen wird auf etwa 14/100.00o Patientenjahre geschätzt. Nach einer Übersichtsstudie von Pretre et al. [21] wurden nach einem stumpfen Thoraxtrauma eine Verletzung des Herzens in $15^{-20} \%$, der Gefäße in $1^{-}$ $17 \%$ der Autopsien gefunden (• Tab. 1, 2). Nach Svennevig et al. [1, 26], die 652 lebend eingelieferte Patienten untersuchten, steigt die Mortalität nach stumpfem Thoraxtrauma in Abhängigkeit von der Zahl der Begleitverletzungen. Sie beträgt

- bei einem Thoraxtrauma allein $2 \%$,

- bei einer zusätzlichen extrathorakalen Verletzung 3\%,

- bei 2 extrathorakalen zusätzlichen Verletzungen $23 \%$ und
- bei im Schockzustand eingelieferten Patienten $38 \%$.

Zusätzliche abdominelle Verletzungen (Mortalität 31\%) und Schädel-Hirn-Traumen (Mortalität 26\%) sind prognostisch besonders ungünstig [27].

\section{Lebensbedrohliche Befunde}

$\mathrm{Zu}$ den akut lebensbedrohlichen Verletzungen bzw. Befunden am Thorax zählen

- Herzbeuteltamponade

- massiver Hämatothorax

- Atemwegsobstruktion

- Spannungspneumothorax

- offener Pneumothorax

Potenziell lebensbedrohlich sind

- Herzkontusion

- (gedeckte) Ruptur der Aorta

- Zwerchfellruptur

- Lungenkontusion

- tracheobronchiale und ösophageale Verletzungen

Frakturen von weniger als 3 Rippen, Sternumfrakturen, der unkomplizierte Hämatothorax und der Befund eines subkutanen Emphysems sind in der Regel nicht lebensbedrohlich $[12,26]$.

\section{Klinische Befunde}

Direkte scharfe Verletzungen der Gefäße gehen in der Regel mit heftigen Blutungen einher. Stumpfe Traumen sind durch Einrisse der Intima oder Media bei noch erhaltener Adventitia charakterisiert [15]. Bei Beteiligung des Herzens kommt es in Abhängigkeit von der Größe des verletz- ten Areals und der Lokalisation zu einer Einschränkung der Pumpfunktion (Myokardkontusion, Perikardtamponade).

Der Unfallmechanismus, der Lokalbefund (Art der Wunde, Blutungsqualität, Schwellung, Hämatom, Prellmarken), Begleitverletzungen (Frakturen, Luxationen) und die nachgeschaltete Durchblutungssituation (Hautkolorit, Pulsstatus, Sensibilität, Motorik) sind für die weitere Diagnostik wegweisend [7].

\section{Erstmaßnahmen und Transfer in die Klinik}

Patienten mit dem Verdacht auf eine größere thorakale Gefäß- oder Herzverletzung sollten ohne Verzögerung in ein Zentrum gebracht werden, welches das gesamte Spektrum an diagnostischen Verfahren und an Behandlungsmöglichkeiten möglichst unter Einschluss der Anwendung der extrakorporalen Zirkulation vorhält. Außerhalb der Klinik ist der Versuch einer lokalen Blutstillung bei intrathorakalen Verletzungen nicht indiziert $[7,15]$.

Die Schockbehandlung muss am Unfallort einsetzen und während des gesamten Transports gewährleistet sein. Zur Minimierung des Blutverlusts sollten subnormale Blutdruckwerte angestrebt werden (so genannte permissive Hypotonie) [29]. Patienten nach Thoraxtrauma können durch hohe Blutverluste im hämorrhagischen Schock, aber auch im stabilen Zustand sein.

Von einer Notfallthorakotomie (im Schockraum) können Patienten in marginalem Zustand profitieren, wenn sie 1. nicht kurz vor der Aufnahme in das

Krankenhaus reanimiert wurden, 


\section{Hier steht eine Anzeige.}

算 Springer 


\begin{tabular}{|llll|}
\hline Tab. 1 Verletzungen des Herzens bei stumpfem Thoraxtrauma & \\
\hline Herzverletzung & & Autopsiestudien [\%] & Klinische Serien [\%] \\
\hline Klappenverletzung & $15-20$ & $16-76$ \\
\hline Koronararterienverletzung & 5 & Fallberichte \\
\hline Kammerruptur & 2 & Fallberichte \\
& & $36-65$ & $0,3-0,9$ \\
\cline { 2 - 4 } & Rechter Vorhof & $10-15$ & $36-65$ \\
\cline { 2 - 4 } & Rechter Ventrikel & $19-32$ & $17-32$ \\
\cline { 2 - 4 } & Linker Vorhof & $1-7$ & $20-31$ \\
\cline { 2 - 4 } & Linker Ventrikel & $17-44$ & $11-15$ \\
\cline { 2 - 4 } & Mehr als 1 & $23-32$ & $6-10$ \\
\hline
\end{tabular}

\section{Tab. 2 Thorakale Gefäßverletzungen bei stumpfem Thoraxtrauma}

\begin{tabular}{lll} 
& Autopsiestudien [\%] & Klinische Serien [\%] \\
Gefäßverletzung & $15-17$ & 4 \\
\hline Aorta ascendens und Bogen & $8-23$ & $0-10$ \\
\hline Aortenisthmus & $70-86$ & $80-90$ \\
\hline Aorta (Hiatus) & $0-14$ & $0-5$ \\
\hline Truncus brachiocephalicus & Nicht angegeben & $20-61$ \\
\hline Karotiden & Nicht angegeben & $0-26$ \\
\hline Aa. subclavia & Nicht angegeben & $12-19$ \\
\hline $\begin{array}{l}\text { Mehr als 1 } \\
\text { Modifiziert nach Pretre u. Chilcott [20] }\end{array}$ & $5-10$ \\
\hline
\end{tabular}

2. bei der Aufnahme einen stabilen Herzrhythmus hatten,

3. hypoton sind,

4. nicht auf andere Wiederbelebungsmaßnahmen ansprechen oder

5. kurz nach der Aufnahme einen Herzstillstand erlitten haben.

Die Überlebenschance beträgt nach stumpfem Thoraxtrauma in solchen Fällen dennoch weniger als $2 \%$, nach penetrierenden Verletzungen $8 \%[9,27]$.

\section{Bildgebende Diagnostik}

Sie sollte, wann immer möglich, durchgeführt werden, um die Indikation zur Thorakotomie stellen zu können. Ein Algorithmus findet sich in $\mathbf{0}$ Abb. 1 [3, $8,15,22]$. Wir empfehlen heute eine thorakale helikale Computertomographie mit Kontrastmittel, sofern die Befunde im Röntgenbild des Thorax auf eine thorakale Verletzung hinweisen, wie bei

- Verbreitertem oberem Mediastinum $(>8 \mathrm{~cm})$

- Verlust der Aortenkontur

- Hämatom im Bereich der linken apikalen Pleura

- Sternum- oder Skapulafraktur
- Fraktur der 1. und/oder 2. Rippe

- Hämatom im Bereich des „thoracic outlet"

- Massivem linkem Hämatothorax

- Fehlen des aortopulmonalen Fensters in der seitlichen Aufnahme

- Verlagerung der Trachea nach vorn (seitliches Bild) oder zur Gegenseite (bei Hämatothorax)

- Abweichung der Magensonde im Ösophagus von der Mittellinie

- Dislozierte Wirbelkörperfrakturen im Thoraxbereich

Steht ein leistungsfähiger Computertomograph in der Notaufnahme ohne Zeitverlust zur Verfügung, kann auch auf eine Röntgenaufnahme des Thorax verzichtet werden.

Unterstützend sind die transthorakale oder transösophageale Echokardiographie (Hämatothorax, Perikardtamponade, Aortendissektion) anzuwenden.

Die Magnetresonanztomographie hat in der Notfalldiagnostik keine Bedeutung.

Die Angiographie kann als unterstützendes Verfahren bei besonderen Fragestellungen angewendet werden, ist aber als Goldstandard von der CT, mit der auch ei- ne dreidimensionale Rekonstruktion der Pathologie erfolgen kann, abgelöst worden. Zur Planung endovaskulärer Therapien ist ebenfalls eine CT vorzuschalten.

\section{Operative Therapie}

\section{Indikationen}

Bei folgenden Konstellationen ist eine Thorakotomie indiziert:

1. mediastinale Durchschussverletzung

2. Herzstillstand im Krankenhaus

3. Perikardtamponade

4. radiologischer Verdacht auf Verletzung eines großen Gefäßes

5. fortbestehender Blutverlust über die Thoraxdränage von initial mehr als 1000-1500 $\mathrm{ml}$ und anschließend mehr als 150-300 $\mathrm{ml}[6,24,27]$.

\section{Zugangswege}

Verfügbar sind: mediane Sternotomie, anterolaterale Thorakotomie, bilaterale, anterolaterale Thorakotomie (mit Sternumdurchtrennung, „clamp shell“), partielle obere Sternotomie („open book“- oder „trap door“-Thorakotomie) und posterolaterale Thorakotomie [5, 27].

Mediane Sternotomie. Sie ermöglicht die optimale Exposition bei Verletzungen des Herzens, der aszendierenden Aorta, der V. brachiocephalica, des Truncus brachiocephalicus und der oberen Hohlvene.

Linksanterolateraler Zugang. Er ist für die offene Reanimation geeignet. Über ihn kann nach Durchtrennung des Sternums und evtl. kontralateraler Erweiterung eine Vielzahl von Operationen im Bereich des Thorax durchgeführt werden.

Partielle obere Sternotomie. Die supraaortalen Äste können an ihrem Ursprung aus der Aorta über eine solche mit Erweiterung der Inzision in den 3. oder 4. Interkostalraum sowie kranial am Vorderrand des M. sternocleidomastoideus bzw. supraklavikular erreicht werden [14].

Linksseitige posterolaterale Thorakotomie. Über sie wird die deszendierende Aorta exponiert. 


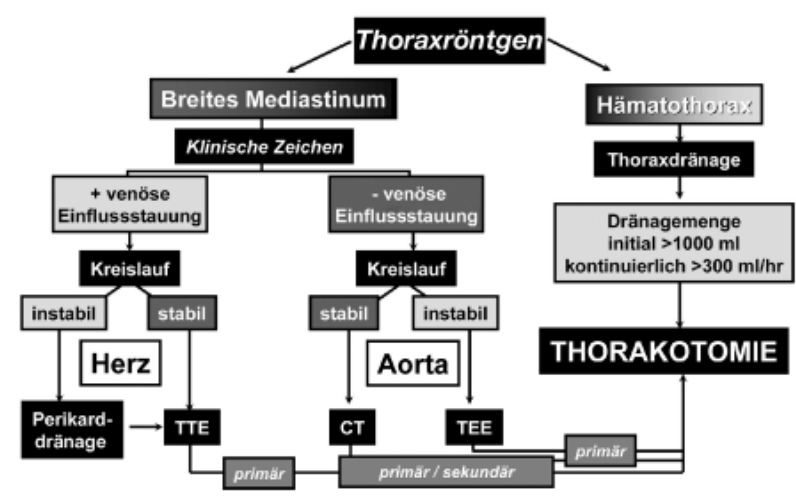

Abb. 1 A Algorithmus für Diagnostik und Therapie bei stumpfem Thoraxtrauma in der Notaufnahme

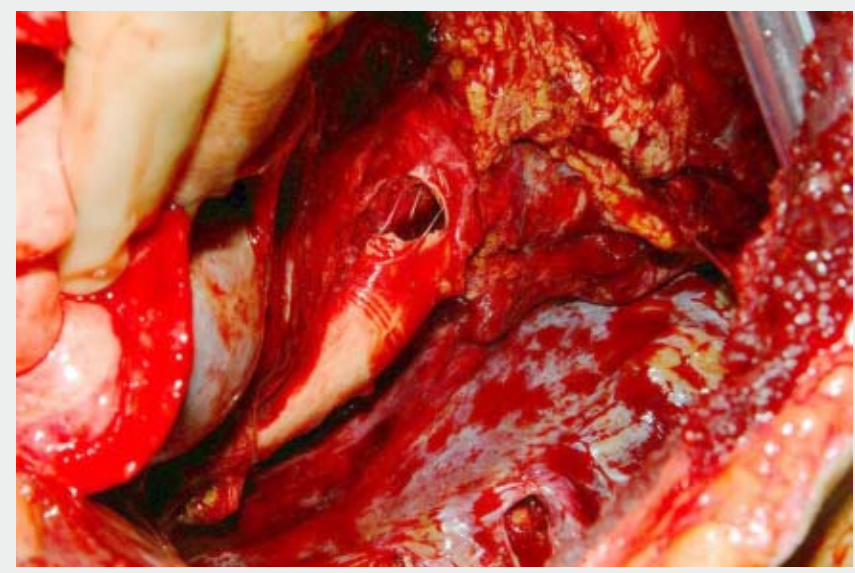

Abb. $2 \Delta$ Aortenruptur an typischer Stelle, exploriert über linksseitige posterolaterale Thorakotomie, polytraumatisierter Patient, in tabula durch Exsanguination bei gleichzeitig vorliegendem abdominellem Trauma mit Leber- und Milzruptur verstorben
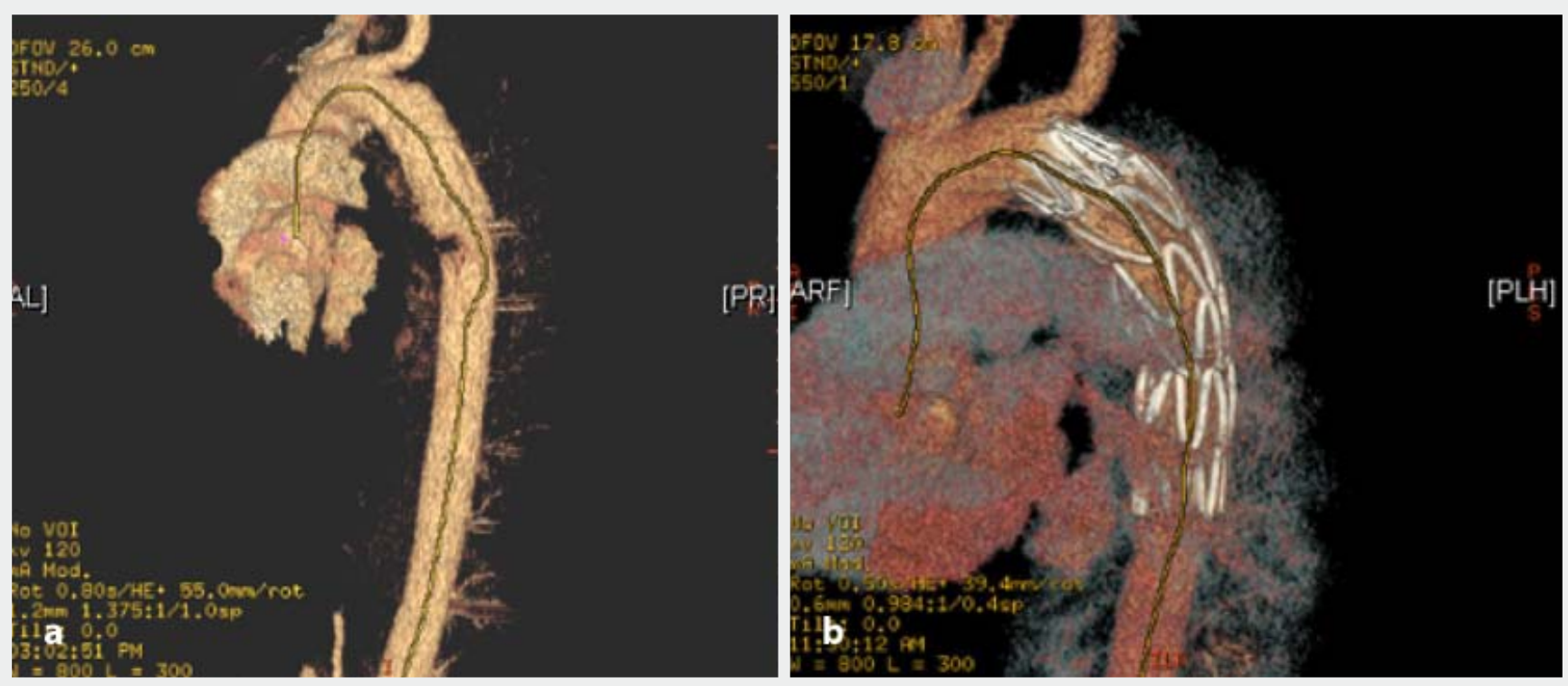

Abb. $3 \Delta$ Computertomographische Rekonstruktion der Aorta, Intimaläsion in der Aorta descendens, gedeckte Ruptur (nicht sichtbar) (a), Patient als Fahrer eines KFZ verunfallt, noch am Unfallort Entwicklung einer Paraplegie, nach endovaskulärer Versorgung mit ummanteltem thorakalem Stentgraft (Metronic Valiant) (b) komplette Rückbildung der Paraplegie, in Rekonstruktion Metallgittersystem gut sichtbar

\section{Spezielle Verletzungen}

\section{Herzkontusion}

Die Diagnose Myokardkontusion ist problematisch zu stellen, da die Befunde nicht einheitlich sind. Im EKG können (aber nicht zwingend) Arrhythmien, Blockbilder oder/und Ischämien dokumentiert werden [19]. Wandbewegungsstörungen durch Kontusion des Herzmuskels und Verletzungen von Herzklappen oder Papillarmuskeln können mit der Echokardiographie nachgewiesen werden. Laborparameter wie die CK-
MB oder der Troponinspiegel einschließlich der Isoenzyme des Troponins sind aufgrund der häufigen muskuloskelettalen Begleitverletzungen oder Nierenfunktionsstörungen meist nicht aussagekräftig. Neuere Untersuchungsmethoden wie die PET sind beschrieben [17]. Ihre Bedeutung für die Routinediagnostik wird wegen des vergleichsweise großen Aufwands wohl gering bleiben.

Die Therapie der Herzkontusion erfolgt konservativ (Analgesie, Oxygenierung, Ruhe) [2].

\section{Herzruptur}

Die Diagnose erfolgt klinisch und durch Echokardiographie (Algorithmus zur Diagnosestellung s. $\bullet$ Abb. 1).

Die Mortalität der lebend das Krankenhaus erreichenden Patienten beträgt auch in spezialisierten Zentren 70\%.

Die Versorgung erfolgt aufgrund des Notfallcharakters überwiegend mit direkter Naht ohne Einsatz der Herz-Lungen-Maschine. Die chirurgische Therapie von perforierenden Herzverletzungen besteht in der Kontrolle der Blutung und Entlastung der Perikardtam- 
Trauma Berufskrankh 2007 - 9 [Suppl 2]: S118-S123

DOI 10.1007/s10039-006-1126-y

c) Springer Medizin Verlag 2006

O. E. Teebken - A. M. Pichlmaier - M. Karck · A. Haverich

\section{Herz- und thorakale Gefäßverletzungen}

\section{Zusammenfassung}

Die Mortalität von Herz- und thorakalen Gefäßverletzungen ist sehr hoch, abhängig von den Begleitverletzungen und der Qualität der Erstversorgung sowie den Möglichkeiten im versorgenden Zentrum. Ätiologie, Inzidenz, diagnostische und therapeutische Algorithmen, operative Zugangswege und Methoden einschließlich der endovaskulären Versorgung von Aortenrupturen werden erläutert. Im Zeitraum von 1997-2004 wurden in der Medizinischen Hochschule Hannover 41 Patienten (39 männlich, 2 weiblich) im Alter von 8-83 Jahren (Mittel 38 Jahre) wegen einer Herz- oder thorakalen Gefäßverletzung versorgt. Die 30-Tage-Mortalität betrug in unserem Kollektiv 9,8\%.

\section{Schlüsselwörter}

Aortenruptur · Herzverletzung · Penetrierende Thoraxverletzung · Stumpfe Thoraxverletzung · Thoraxtrauma

\section{Treatment of major injuries of the heart and thoracic vessels}

\begin{abstract}
Injuries to the heart and the intrathoracic vessels involve a high mortality rate. The outcome depends on the concomitant injuries sustained and the quality of primary care, and also on the options available in the care centre. Aetiology, incidence, diagnostic and therapeutic algorithms, and surgical approaches and methods, including endovascular repair of aortic ruptures, are described. At the Hanover Medical School 41 patients (39 male, 2 female) aged 8-83 (mean 38 ) years were treated for penetrating heart or vascular injuries from 1997 to 2004. The 30-day mortality was $9.8 \%$.
\end{abstract}

\section{Keywords}

Aortic rupture - Myocardial injury · Penetrating chest trauma - Blunt chest trauma - Thoracic injury ponade nach Thorakotomie. Wir empfehlen die mediane Sternotomie als $\mathrm{Zu}$ gang. Grundsätzlich, je nach Erfahrung des Chirurgen und beim Fehlen entsprechender Instrumente, kann auch eine laterale Thorakotomie angewendet werden. Eine vital bedrohliche Perikardtamponade kann über einen subxyphoidalen Zugang oder über eine anterolaterale Thorakotomie mittels inferiorer Perikardiotomie entlastet werden.

\section{Koronarien und Herzklappen}

Die Diagnose wird echokardiographisch, bei Koronarienverletzungen ggf. unter Hinzuziehung der Koronarangiographie, gestellt [10]. Die chirurgische Therapie erfolgt meist mit aufgeschobener Dringlichkeit unter Einsatz der extrakorporalen Zirkulation je nach Befund als Herzklappenrekonstruktion, Herzklappenersatz oder operative Myokardrevaskularisation. In weniger schweren Fällen ist auch eine konservative Behandlung möglich.

\section{Aorta ascendens und Aortenbogen}

Im Gegensatz zur Aorta descendens wird die Aorta ascendens meist im Rahmen penetrierender Verletzungen und nicht durch stumpfe Traumen geschädigt. Abgesehen von penetrierenden Wunden der aszendierenden Aorta und des Aortenbogens, welche ggf. nach tangentialer Ausklemmung mit direkter Naht versorgt werden können, ist in der Regel der Einsatz der extrakorporalen Zirkulation notwendig. Die Therapie besteht dann im prothesialen Ersatz des Gefäßabschnitts.

\section{Truncus brachiocephalicus}

Verletzungen desselben führen zu einer im Röntgen sichtbaren Verbreiterung des oberen Mediastinums und nicht selten zu einer Blutung in die Pleura. Die Rekonstruktion erfolgt direkt oder durch prothesialen Ersatz in End-zu-End- oder Endzu-Seit-Technik. Großer Wert ist auf die adäquate Entlüftung und die Entfernung von Debris vor Wiedereröffnung der zerebralen Zirkulation zu legen. Die Überlebensrate beträgt mehr als 90\% bei Patienten, die kreislaufstabil und ohne zere- brale Verletzungen das Krankenhaus erreicht haben.

\section{Aorta descendens}

Penetrierende Wunden derselben können abhängig von ihrer Größe und Lokalisation mit einer lateralen Aortoraphie, einer Patchplastik oder auch einem prothesialen Ersatz behandelt werden. Typischerweise führen stumpfe Traumen zu einer Läsion distal des Lig. arteriosum (D Abb. 2) [4, 25]. Die Versorgung erfolgt über eine linksposterolaterale Thorakotomie, je nach Gegebenheiten mit oder ohne Hilfe der extrakorporalen Zirkulation. In $80 \%$ der Fälle wird ein Dacroninterpositionsgraft verwendet. Die Mortalität beträgt beim offen-chirurgischen Vorgehen $15-28 \%$. Durch eine systemische Heparinisierung steigt das Risiko für schwere Blutungen, insbesondere für Patienten mit schweren Begleitverletzungen [27].

Für gedeckte Rupturen und traumatische Dissektionen, bei denen keine Notwendigkeit zur kurzfristigen Versorgung besteht (,aufgeschobene Dringlichkeit“), ist aktuellen Untersuchungen zufolge die endovaskuläre Versorgung mittels thorakalen ummantelten Stents (- Abb. 3) mit einer im Vergleich zum offenen chirurgischen Vorgehen geringeren Mortalität (etwa 6\%) und Komplikationsrate, insbesondere Paraplegierate, verbunden [16, $18,23]$.

\section{A. subclavia}

Verletzungen führen zu einem Hämatothorax oder einem Hämatom im Bereich der oberen Thoraxapertur $[11,13,15]$. Die Kontrolle der Arterie und ihrer Äste ist über eine partielle obere Thorakotomie mit Verlängerung der Inzision nach supraklavikular möglich. Die Rekonstruktion kann mittels Prothese oder autologer Vene erfolgen. $\mathrm{Zu}$ beachten ist, dass die Wand der A. subclavia äußerst fragil ist. Aufgrund der Koinzidenz von Verletzungen der A. subclavia und des Plexus brachialis sollte nach Möglichkeit ein präoperativer fachneurologischer Status zur Abgrenzung von traumatisch vorbestehenden Komplikationen von potenziell operationsbedingten erhoben werden7 


\section{Intrathorakale Venen}

Verletzungen der V. cava, V. brachiocephalica oder V. azygos, in der Regel in Zusammenhang mit penetrierenden Stichverletzungen, sind sehr schwer zu kontrollieren [28]. Nach der Thorakotomie ist eine ausreichende Blutungskontrolle meist durch eine manuelle Kompression zu erreichen. Bei der Verwendung von Retraktoren zur Verbesserung der Exposition oder beim Einsatz von Gefäßklemmen ist zu beachten, dass dadurch die Schädigung iatrogen vergrößert und die Rekonstruktion zusätzlich erschwert werden können. Die V. azygos kann ohne negative Folgen ligiert werden. Zur Rekonstruktion der V. cava bieten sich autologe Venenpatches, bovines glutaraldehydfixiertes Perikard oder im Fall eines Ersatzes eine Dacron- oder ringarmierte PTFE-Prothese an. Aufgrund des Notfallcharakters verbieten sich aufwändige Rekonstruktionen mit Spiralvenengrafts in den meisten Fällen.

\section{Eigene Ergebnisse beim penetrierenden Thoraxtrauma}

Im Zeitraum von 1997-2004 wurden in der Medizinische Hochschule Hannover 41 Patienten (39 männlich, 2 weiblich) im Alter von 8-83 Jahren (Mittel 38 Jahre) wegen einer Herz- oder thorakalen $\mathrm{Ge}$ fäßverletzung versorgt. 8 Patienten hatten eine Schusswunde und 33 andere penetrierende Verletzungen. 8 Patienten wurden konservativ behandelt und 33 operiert. Dabei wurden 15 über eine mediane Sternotomie (mehrheitlich Herzverletzungen) versorgt, 17 wurden lateral thorakotomiert, und ein Patient erhielt lediglich eine Lavage und ein oberflächliches Débridement des Einstichkanals. 7 Patienten wurden zusätzlich wegen Begleitverletzungen laparotomiert. Die 30-Tage-Mortalität betrug in unserem Kollektiv $9,8 \%$.

\section{Fazit für die Praxis}

Intrathorakale Gefäßverletzungen und
Verletzungen des Herzens sind mit einer
sehr hohen Mortalität verbunden. Auf-
grund der heutigen Subspezialisierung
stehen nicht in jedem Krankenhaus, ins- besondere im Bereitschaftsdienst, Spezialisten für Trauma-, Herz-, Thorax- oder Gefäßchirurgie zur Verfügung. Im Fall einer thorakalen Gefäßverletzung oder einer Verletzung des Herzens sollte der Transport daher primär in ein Zentrum erfolgen, in dem entsprechende Spezialisten zur Verfügung stehen und die Versorgung mit Hilfe der extrakorporalen Zirkulation oder eine endovaskuläre Stentimplantation (Aorta-descendens-Verletzung) möglich sind. Eine Ausnahme davon stellt nur die Indikation zur Notthorakotomie dar.

\section{Korrespondierender Autor \\ PD Dr. O. E. Teebken}

Klinik für Thorax-, Herz- und Gefäßchirurgie, Medizinische Hochschule Hannover; Carl-Neuberg-Straße 1, 30625 Hannover teebken.omke@mh-hannover.de

Interessenkonflikt. Es besteht kein Interessenkonflikt. Der korrespondierende Autor versichert, dass keine Verbindungen mit einer Firma, deren Produkt in dem Artikel genannt ist, oder einer Firma, die ein Konkurrenzprodukt vertreibt, bestehen. Die Präsentation des Themas ist unabhängig und die Darstellung der Inhalte produktneutral.

\section{Literatur}

1. Acosta JA, Yang JC, Winchell RJ et al. (1998) Lethal injuries and time to death in a level I trauma center. J Am Coll Surg 186: 528-533

2. Bernardis V, Kette F, Blarasin L et al. (2004) Isolated myocardial contusion in blunt chest trauma. Eur J Emerg Med 11: 287-290

3. Britt LD, Weireter LJ, Cole FJ (2001) Newer diagnostic modalities for vascular injuries: the way we were, the way we are. Surg Clin North Am 81: 1263-1279

4. Carter Y, Meissner M, Bulger E et al. (2001) Anatomical considerations in the surgical management of blunt thoracic aortic injury. J Vasc Surg 34: 628633

5. Degiannis E, Bonanno F, Titius W et al. (2005) [Treatment of penetrating injuries of neck, chest and extremities]. Chirurg 76: 945-958

6. Demetriades D (1997) Penetrating injuries to the thoracic great vessels. J Card Surg 12: 173-180

7. Gefäßchirurgie VdDGf (1998) Leitlinien zur Diagnostik und Therapie in der Gefäßchirurgie. Deutscher Ärzteverlag, Köln, S 123-127

8. Halter G, Orend KH (2005) [Thoracic, abdominal, and pelvic vascular injuries]. Chirurg 76: 411-424

9. Heineck J, Jacobi TH, Saeger HD, et al. (2005) Thoraxtrauma. Trauma Berufskrankh 7: S202-S206

10. Hibino N, Tsuchiya K, Sasaki H et al. (2003) Delayed presentation of injury to the sinus of valsalva with aortic regurgitation resulting from penetrating cardiac wounds. J Cardiac Surg 18: 236-239

11. Holdgate A, Dunlop S (2005) Review of branch aortic injuries in blunt chest trauma. Emerg Med Australas 17: 49-56
12. Kuhne $C A$, Ruchholtz S, Voggenreiter G et al. (2005) [Traumatic aortic injuries in severely injured patients]. Unfallchirurg 108: 279-287

13. Makaroun MS, Dillavou ED, Kee ST et al. (2005) Endovascular treatment of thoracic aortic aneurysms: results of the phase II multicenter trial of the GORE TAG thoracic endoprosthesis. J Vasc Surg 41: 1-9

14. Mclntyre WB, Ballard JL (1998) Cervicothoracic vascular injuries. Semin Vasc Surg 11: 232-242

15. Nzewi O, Slight RD, Zamvar V (2006) Management of blunt thoracic aortic injury. Eur J Vasc Endovasc Surg 31: 18-27

16. Orend KH, Kotsis T, Scharrer-Pamler R et al. (2002) Endovascular repair of aortic rupture due to trauma and aneurysm. Eur J Vasc Endovasc Surg 23: 61-67

17. Pai M (2006) Diagnosis of myocardial contusion after blunt chest trauma using 18F-FDG positron emission tomography. Br J Radiol 79: 264-265

18. Peterson BG, Matsumura JS, Morasch MD et al. (2005) Percutaneous endovascular repair of blunt thoracic aortic transection. J Trauma 59: 10621065

19. Pizzo VR, Beer I, Cleva R de et al. (2005) Intermittent left bundle branch block (LBBB) as a clinical manifestation of myocardial contusion after blunt chest trauma. Emerg Med J 22: 300-301

20. Pretre R, Chilcott M (1997) Blunt trauma to the heart and great vessels. N Engl J Med 336: 626632

21. Pretre R, Chilcott M, Murith N et al. (1997) Blunt injury to the supra-aortic arteries. Br J Surg 84: 603609

22. Rieger M, Sparr H, Esterhammer R et al. (2002) [Modern CT diagnosis of acute thoracic and abdominal trauma]. Anaesthesist 51: 835-842

23. Rousseau H, Dambrin C, Marcheix B et al. (2005) Acute traumatic aortic rupture: a comparison of surgical and stent-graft repair. J Thorac Cardiovasc Surg 129: 1050-1055

24. Segers $P$, Van Schil $P$, Jorens $P$ et al. (2001) Thoracic trauma: an analysis of 187 patients. Acta Chir Belg 101: 277-282

25. Stiles QR, Cohlmia GS, Smith JH et al. (1985) Management of injuries of the thoracic and abdominal aorta. Am J Surg 150: 132-140

26. Svennevig JL, Bugge-Asperheim B, Geiran OR et al. (1986) Prognostic factors in blunt chest trauma. Analysis of 652 cases. Ann Chir Gynaecol 75: 8-14

27. Teebken OE, Haverich A (2005) [Diagnostics and treatment of thoracic vascular injuries]. Zentralbl Chir 130: W14-22

28. Wall MJ Jr, Mattox KL, Debakey ME (2006) Injuries to the azygous venous system. J Trauma 60: 357362

29. Ziegenfuss T (1996) [Emergency management of polytrauma patients]. Zentralbl Chir 121: 924-942 